FERMILAB-TM-2371-DO

October 2006

\title{
Accelerator/Experiment Operations - FY 2006
}

\author{
S. Brice, J. Conrad, D. Denisov, G. Ginther, S. Holmes, C. James, W. Lee, \\ W. Louis, C. Moore, R. Plunkett, R. Raja, E. Ramberg, W. Sakumoto, \\ L. Stutte, R. Van de Water, P. Wilson, T. Wyatt, T. Yasuda
}

Edited by J. Appel

This Technical Memorandum (TM) summarizes the Fermilab accelerator and experiment operations for FY 2006. It is one of a series of annual publications intended to gather information in one place. In this case, the information concerns the FY 2006 Run II at the Tevatron Collider, the MiniBooNE experiments running in the Booster Neutrino Beam in neutrino and antineutrino modes, MINOS using the Main Injector Neutrino Beam (NuMI), and SY 120 activities.

Each section was prepared by the relevant authors, and was somewhat edited for inclusion in this summary.

$\underline{\text { Accelerator Operations }}$ (S. Holmes)

\section{$\underline{\text { Tevatron Collider }}$}

Tevatron Collider operations spanned the FY 2005-2006 boundary without interruption, and continued until the initiation of the FY 2006 shutdown on February 26, 2006. The shutdown was completed on schedule, and operations resumed with the first luminosity delivered on June 12 - approximately one week ahead of schedule. For FY 2006, the average of CDF and DZero initially-reported luminosity totaled $621.0 \mathrm{pb}^{-1}$ (Figure 1), 78\% of the design goal. This was achieved over a total of 3647 store hours, $96 \%$ of the design goal. These FY 2006 numbers come from the Laboratory's Monday-to-Monday reporting periods (defined as 00:00 on October 3, 2005 to 00:00 on October 2, 2006. Total integrated luminosity for Run II now stands at $1.896 \mathrm{fb}^{1}$. All values above relate to on-line measurements (see below) as reported to the DOE. In their sections, CDF and DZero report their off-line corrected luminosity values.

Highlights for the year include:

- A record initial luminosity (average of CDF and DZero initially-reported values) of $22.9 \times 10^{31} \mathrm{~cm}^{-2} \mathrm{sec}^{-1}$ on September $20-\mathrm{a} 75 \%$ increase over the record at the end of FY 2005.

- A record weekly delivered luminosity (average of CDF and DZero initially-reported values) of $33.3 \mathrm{pb}^{-1}$ over the period August 28-September 3-a 57\% increase over the record at the end of FY 2005. 
- Full integration of electron cooling into the antiproton complex, allowing the establishment of "Recycler only" shots as the standard operational mode.

- Improvements to the antiproton stacking rate. The stacking rate rose to approximately $20 \times 10^{10}$ /hour over the year, a roughly 20\% improvement over FY 2005.

- Reduction of the time required to transfer antiprotons from the Accumulator to the Recycler. These transfers now routinely take about 25 minutes, reduced from about 45 minutes last year.

- Operation of NuMI in parallel with the collider program. A total of $1.0 \times 10^{20}$ protons were delivered to the NuMI target for the year. The total of delivered protons to NuMI/MINOS stood at $1.7 \times 10^{20}$ at the end of the fiscal year.

- Operation of the Booster Neutrino Beam in parallel with the collider and NuMI programs. A total of $1.7 \times 10^{20}$ protons were delivered to the MiniBooNE target for the year. The total of delivered protons to MiniBooNE stood at $8.0 \times 10^{20}$ at the end of the fiscal year.

- Operation of the $120 \mathrm{GeV}$ fixed-target program (SY120) in parallel with antiproton stacking and neutrino operations.

FY2006 Integrated Luminosity

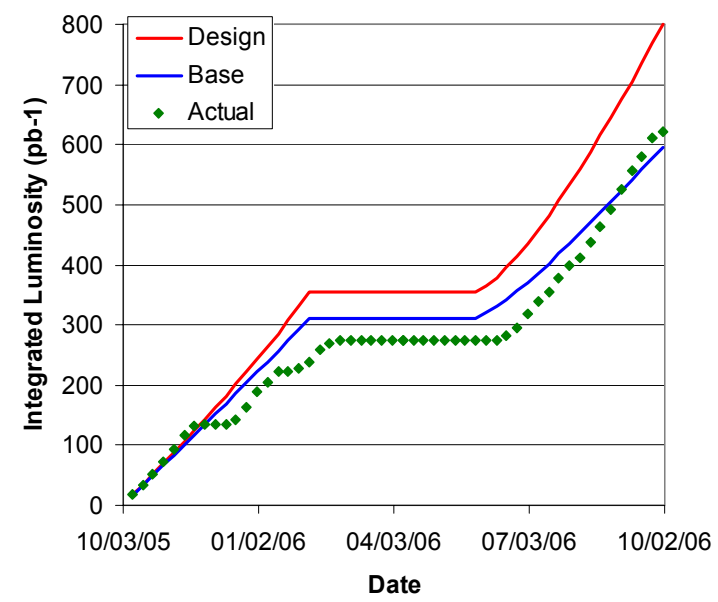

Figure 1. FY 2005 integrated luminosity.

The stage was set for the improvements above by the successful commissioning of the electron cooling system in the Recycler in the fall of 2005, and by improvements incorporated into the accelerator complex during the spring 2006 shutdown.

Also of note, at the end of the year DZero completed analysis of their new luminosity monitoring system and made an adjustment, effective 10/1/06, that raised their reported luminosity by $12 \%$. Similarly, CDF identified an off-line calibration upwards of $1.9 \%$ earlier. These changes are being implemented for FY 2007 reporting. The implication is that all $C D F / D Z$ Zero average luminosity numbers (e.g., peak and integral) reported prior to FY 2007 are underreported by $\sim 7 \%$. 


\section{FY 2006 Shutdown}

The FY 2006 shutdown began on February 26, 2006. This shutdown had been delayed from the original August 2005 schedule to assure readiness for the DZero silicon installation, and for treatment to kill zebra mussels in the industrial cooling water (ICW) system. The shutdown was officially completed on May 31, 2006 coincident with the commencement of Tevatron beam commissioning activities. The first luminosity was generated on June 12. Summarized below is the final status of major shutdown activities aimed at improved performance.

\begin{tabular}{|c|c|c|}
\hline Machine/Activity & Goal & Status \\
\hline \multicolumn{3}{|l|}{ Booster } \\
\hline $\begin{array}{l}\text { New } 400 \mathrm{MeV} \text { injection girder and } \\
\text { ORBUMP installation }\end{array}$ & Reduce losses during Booster injection & Complete \\
\hline $\begin{array}{l}\text { New MI-8 line dump; remove L13 extraction } \\
\text { system removal }\end{array}$ & $\begin{array}{l}\text { Reduce losses during Booster acceleration } \\
\text { cycle }\end{array}$ & Complete \\
\hline \multicolumn{3}{|l|}{ Antiproton Source } \\
\hline New AP-2 correctors & Increase AP-2 aperture (antiproton yield) & Complete \\
\hline Debuncher kicker motorized stands & Increase Debuncher aperture (antiproton yield) & Complete \\
\hline AP30 SO cord remediation & Remediate OSHA violation & Complete \\
\hline \multicolumn{3}{|l|}{ Main Injector } \\
\hline $\begin{array}{l}\text { New large-aperture quadrupoles at injection/ } \\
\text { extraction areas }\end{array}$ & Reduce losses & Complete \\
\hline \multicolumn{3}{|l|}{ Tevatron } \\
\hline New separators & Improve helix flexibility & Complete \\
\hline $\begin{array}{l}\text { Remeasurement and unrolling of misaligned } \\
(<1 \mathrm{mrad}) \text { magnets }\end{array}$ & Reduce global coupling & Complete \\
\hline $\begin{array}{l}\text { Completion of smart bolt shimming ( } 244 \\
\text { magnets) }\end{array}$ & Reduce global coupling & $\begin{array}{l}\text { Complete. This completes } \\
\text { shimming of all Tevatron } \\
\text { dipoles, begun in } 2003 \text {. }\end{array}$ \\
\hline Vacuum upgrades at A0 and D0 & Improve reliability & Complete \\
\hline Tevatron Electron Lens (TEL) II Installation & Compensate for beam-beam effects & Complete \\
\hline Tevatron Electron Lens (TEL) I Repair & $\begin{array}{l}\text { Compensate for beam-beam effects and do } \\
\text { abort gap cleaning }\end{array}$ & Complete \\
\hline $\begin{array}{l}\text { Kautzky valve components replacement } \\
(\sim 1500)\end{array}$ & Improve reliability & Complete \\
\hline \multicolumn{3}{|l|}{ NuMI } \\
\hline Horn 2 water leak repair & Improve operability & Complete \\
\hline \multicolumn{3}{|l|}{ Tritium Remediation } \\
\hline NuMI Target Hall dehumidification system & Reduce tritium source & Complete \\
\hline Target Hall chiller repair & Reduce tritium source & Complete \\
\hline ICW direct connect NuMI-CUB & Reduce tritium source & Complete \\
\hline MI Pond C-D manhole repair & Protect creeks & Complete \\
\hline Booster sump discharge rerouting & Protect creeks & Complete \\
\hline AP-0 sump discharge rerouting & Protect creeks & Complete \\
\hline \multicolumn{3}{|l|}{ Infrastructure Maintenance } \\
\hline Zebra mussel kill & Improve ICW system reliability & Complete \\
\hline Power pole replacement & Improve reliability & $\begin{array}{l}\text { Complete. Pole was } \\
\text { identified during shutdown } \\
\text { as incipient failure. }\end{array}$ \\
\hline Feeder Maintenance & Improve reliability & Complete \\
\hline
\end{tabular}


Commentary on FY 2006 Performance

The median collider initial luminosity over the final six weeks of FY 2006 was $16.8 \times 10^{31}$

$\mathrm{cm}^{-2} \mathrm{sec}^{-1}$, a $63 \%$ increase over the end of FY 2005, and $84 \%$ of the design goal of $19.9 \times 10^{31}$ $\mathrm{cm}^{2} \mathrm{sec}^{-1}$. Improved performance over the course of the year derived from a number of sources, some anticipated and some not. The most significant factors include:

\section{More protons in collision}

The number of protons in collision increased roughly 5-10\% compared to a year ago. Two factors allowed pushing the proton intensity up:

- The installation of new separators during the spring shutdown enabled the implementation of a new $150 \mathrm{GeV}$ helix which significantly improved proton lifetime in the Tevatron during the antiproton loading process.

- Booster performance improved following the installation of a new injection system and the removal of the old L13 extraction system during the spring shutdown.

2. More antiprotons in collision, accompanied by good emittances

The number of antiprotons in collision increased by roughly $50 \%$ over the last year. Primary contributors were:

- The full integration of electron cooling has allowed storing up to $400 \times 10^{10}$ antiprotons in the Recycler with longitudinal and transverse emittances appropriate for generating good luminosity. All shots to the Tevatron now rely entirely on antiprotons transferred from the Recycler.

- The implementation of the "momentum mining" technique allows extraction of $98 \%$ of the antiprotons in the Recycler for utilization in the Tevatron. However, during the initial implementation, poor lifetimes and transverse emittance growth during the mining process limited the early effect of this improvement.

- Near the end of the year, an adjustment to the tune working point in the Recycler greatly improved beam lifetime and significantly reduced emittance growth during the mining process. Following implementation of the new working point, luminosity in the Tevatron rose roughly $25 \%$.

- Over the year, the antiproton stacking rate was improved to roughly $20 \times 10^{10} /$ hour. The primary drivers were improvements to the AP2 and Debuncher apertures along with an upgrade of the rotating target. Improved stacking was accompanied by a significant reduction in the interruption to stacking required for Accumulator-to-Recycler transfers (25 minutes vs. 45 minutes). As a result, operation with more frequent transfers has been implemented, limiting the maximum stack in the Accumulator to about $60 \times 10^{10}$ antiprotons. The result is a higher average stacking rate (by roughly $20 \%$ ).

\section{Improved luminosity lifetime}

The installation of new separators in the Tevatron during the spring shutdown enabled the implementation of a new collision helix. The new helix has improved the luminosity lifetime with a resultant increase of about $10 \%$ in integrated luminosity for the same initial luminosity. 
The primary shortfalls in FY 2006 were related to reliability problems early in the year, and to the antiproton stacking rate. For the year, useful luminosity was available for an average of 101 hours per scheduled week of operations. This was down somewhat from the 107 hours/week averaged in FY 2005. The major contributor to the shortfall was unscheduled downtime related to three separate failures of superconducting magnets in the Tevatron prior to the spring shutdown (one failure is budgeted per year). In total, these three events resulted in the loss of six weeks of running, out of the 36 weeks scheduled for the year. Two of the magnet failures had identical causes during quenches (a particular component in relief valves). All such valves $(\sim 1500)$ had these potentially failing components replaced during the spring shutdown. Reliability after the shutdown was greatly improved, with an average of 120 hours of luminosity per week.

The antiproton stacking rate is the primary remaining key to performance in the Tevatron. The stacking goals established in the spring of 2006 were $25 \times 10^{10}$ antiprotons/hour by September and $30 \times 10^{10}$ /hour by February (2007). Achievement of these goals would allow meeting the long-term collider goal of $8 \mathrm{fb}^{-1}$ by the end of FY 2009. Currently, $20 \times 10^{10} /$ hour is achieved when running well. As a point of reference, the $30 \times 10^{10}$ /hour goal requires: 1) delivery of $8 \times 10^{12}$ protons onto the antiproton production target every 2.2 seconds; and 2) maintaining a production efficiency of $23 \times 10^{-6}$ in the Antiproton Source. Currently, operations are with about $8.0 \times 10^{12}$ protons on target, at a 2.2-2.4 second cycle time, and a production efficiency of about $16 \times 10^{-6}$. The critical elements for achieving the goals are: improved AP2 aperture, improvements to the bandwidth of the core cooling system in the Accumulator, and good Debuncher-toAccumulator transfer efficiency. A (new) task force is pursuing this. The production efficiency through the Debuncher is measured to be in the range $22-23 \times 10^{-6}$. So attention is focused back onto the Debuncher-to-Accumulator transfer and stack-tail cooling performance. Study time aimed at stacking rate improvements will be a continuing feature of operations in FY 2007.

Antiproton availability remains the most important variable determining the luminosity of any particular store. Experience at the end of the year is that luminosities in the range 17$22 \times 10^{31} \mathrm{~cm}^{-2} \mathrm{sec}^{-1}$ are sustainable with a $17 \times 10^{10}$ /hour stacking rate as long as stores remain in the Tevatron for 24-30 hours. For the balance of Run II, the luminosity is expected to be roughly proportional to the achieved stacking rate.

\section{Current Vulnerability/Reliability Issues}

Linac tubes: This long-term vulnerability issue was largely addressed in FY 2006. The strategy of building up a two-year inventory (12 tubes) was completed. This provides the time to develop an alternative source of $\mathrm{rf}$ power for the linac if needed. However, all indications are that the manufacturer will remain a viable source of tubes into the future. 
Status relative to the FY 2006 Plan

Planned and actual performance for FY 2006:

\begin{tabular}{|c|c|c|c|}
\hline & Base Profile & $\underline{\text { Design Profile }}$ & Actual \\
\hline Median Initial Luminosity $\left(\mathrm{cm}^{-2} \mathrm{sec}^{-1}\right)$ & $10.7 \times 10^{31}$ & $19.9 \times 10^{31}$ & $16 . \overline{8 \times 10^{31}}$ \\
\hline Protons/bunch & $250 \times 10^{9}$ & $260 \times 10^{9}$ & $264 \times 10^{9}$ \\
\hline Pbars/bunch & $38 \times 10^{9}$ & $60 \times 10^{9}$ & $56 \times 10^{9}$ \\
\hline Effective Emittance $(\pi \mathrm{mm}-\mathrm{mr})$ & 16 & 16 & 18 \\
\hline Beta at the IP $(\mathrm{cm})$ & 28 & 28 & 28 \\
\hline Hourglass Factor & 0.65 & 0.65 & 0.68 \\
\hline Zero Stack Stacking Rate $\left(\times 10^{10} /\right.$ hour $)$ & 16 & 30 & 17 \\
\hline Integrated luminosity for the period $\left(\mathrm{pb}^{-1}\right)$ & 107.9 & 184.6 & 157.8 \\
\hline FY 2006 integrated luminosity $\left(\mathrm{pb}^{-1}\right)$ & 595.9 & 800.2 & 621.0 \\
\hline FY 2006 integrated store hours (hours) & 3636 & 3780 & 3647 \\
\hline
\end{tabular}

* "Base" and "Design" correspond to end-of-year goals. "Actual" corresponds to simultaneous performance on the median store of the last six weeks of FY 2006.

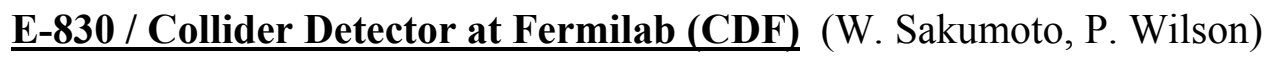

During FY 2006, a total integrated luminosity of $665 \mathrm{pb}^{-1}$ (off-line value corrected upwards by $1.9 \%$ ) was delivered to CDF, of which $544 \mathrm{pb}^{-1}$ was recorded to mass storage. The total data collection efficiency was $82 \%$. The losses of efficiency come from deadtime associated with trigger acceptance, operational inefficiencies (e.g. starting and stopping runs), and downtime from detector problems. By the end of FY 2006, the experiment had been delivered $2 \mathrm{fb}^{-1}$ in Run II (Figure 2). CDF handled the increasing record instantaneous luminosities delivered by the Tevatron without throttling back any high-priority physics triggers. This is due to the timely completion and prompt integration into daily data-taking operations of CDF Run IIb Upgrade Project components. In FY 2005, a faster Level 2 trigger decision system and the hardware event builder system (together tripling the bandwidth into the Level 3 farms) were put into operation.

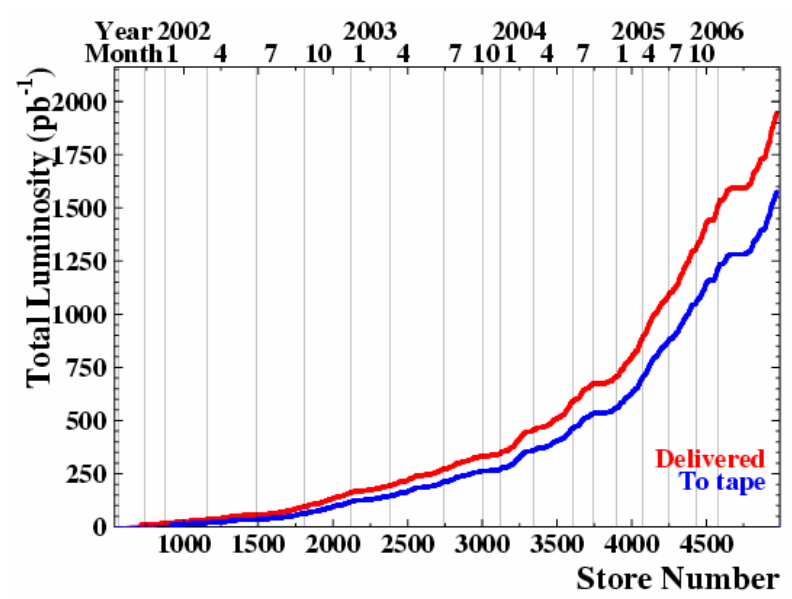

Figure 2. Luminosity (as reported on-line) acquired by CDF in fiscal years 2001-06. 
Other parts of the CDF Run IIb Upgrade Project were put on a fast-track for completion in FY 2006. The time-to-digital converters (TDCs) for the central drift chamber were removed from the detector in small batches, modified for higher-speed readout, and then re-installed. The limited supply of spare TDC boards forced this small-batch installation strategy. The installation of modified boards was completed in December 2005. The upgrade of the silicon vertex trigger (SVT) was installed in three stages, with the first two commissioned in FY 2005, and the third in the first half of FY 2006. The full system was operational in February 2006. The upgraded SVT has higher-capacity pattern memory cards, which allow for a larger number of track patterns and finer track roads. New track-fitting hardware provides faster processing of tracks. The third stage involved buffer memory cards, which were required to fully utilize the expanded pattern memory. The upgraded SVT provides displaced track vertexing at the trigger level with significantly reduced execution time. Also, the Level 3 farm was upgraded with new, faster processors, and was made operational in May 2006. The upgraded Level 3 farm has a processing power of $2.5 \mathrm{THz}$.

The final component of the Run IIb Upgrade Project, the stereo track trigger, needed for running at instantaneous luminosities in excess of $200 \times 10^{30} \mathrm{~cm}^{-2} \mathrm{sec}^{-1}$, was nearing completion at the end of the fiscal year. This upgrade finds track segments in stereo layers of the central tracker, and associates them with axial tracks from the existing Level 1 track trigger processor to reduce the rate of fake tracks. There are two components to this upgrade: The Level 1 and Level 2 paths. The Level 1 path confirms that the axial track has corresponding segments in the stereo layers. This confirmation is available for use in both the Level 1 and Level 2 trigger decisions and will be operational in October 2006. The Level 2 path provides the 3-dimensional track helix to the Level 2 trigger decision system, and will be operational in January 2007.

The CDF Run IIb Upgrade Project met its project completion milestone in January 2006. Not included in the project is an upgrade of the CDF data logger, which began in October 2005. The logger system takes events from the Level 3 farms, buffers them onto local disks, and then puts them onto tape. The logger used in the past had hardware and software components that are obsolete and without maintenance support. The new data logger has a bandwidth of $80 \mathrm{MB} / \mathrm{sec}$, and will no longer be a limitation to sustained data-taking at high instantaneous luminosities. This upgrade will be operational in November 2006.

These upgrades have ensured that the CDF detector has run well and will continue to run well at the highest instantaneous luminosities the Tevatron delivers. There is an ongoing parallel effort of trigger table improvements to utilize the improvements in the CDF data-taking system and to run efficiently at high instantaneous luminosities without significant reduction in datataking efficiency of CDF's high-priority physics triggers.

CDF utilized the accelerator shutdown which began at the end of February to prepare for efficient running without any major shutdowns through 2009. Repairs were made to several detector components, along with repairs and preventive maintenance on detector infrastructure. In addition, the Tevatron made changes directed at improved operation for CDF: 
- Moved the Tevatron beam so that it is better aligned and centered in the CDF detector. The detector has been slowly sinking since 2001, but this covers movement through 2009.

- Addition of a Tevatron collimator at A48 to better protect CDF from beam incidents.

After the shutdown, the Tevatron delivered $371 \mathrm{pb}^{-1}$ to CDF (again, off-line corrected value). During this period, CDF operated with $83 \%$ data-taking efficiency. The Run IIb upgrades were nearly all in operation at the end of FY 2006. They have already significantly improved the datataking capacity of CDF.

$\underline{\text { E-823 / DZero (DZero) }}$ (D. Denisov, G. Ginther, W. Lee, L. Stutte, T. Wyatt, T. Yasuda)

The accelerator complex was in routine operation at the start of FY 2006, and the DZero detector was running reliably and efficiently. Between October 1, 2005 and the start of the 2006 shutdown on February 22, 2006, DZero recorded data with an average efficiency of $86.5 \%$ while preparing for installation and commissioning of a significant suite of trigger, data acquisition, and tracking detector upgrades.

To effectively prepare for the large increases in instantaneous and integrated luminosity anticipated between FY 2006 and FY 2009, DZero designed and produced a suite of upgrades to the trigger system to provide increased trigger rejection. Where feasible, either tests of vertical slices or full systems tests of these trigger elements were performed prior to the 2006 shutdown. Improvements to the trigger were installed and technically commissioned during the 2006 shutdown. The Level 1 calorimeter trigger electronics was replaced in its entirety, to provide sharper turn-on curves and enhanced flexibility in electron, tau, and jet object identification. The electronic components that implement the Level 1 deadtimeless charged-track trigger based upon the information from the scintillating (central) fiber tracker (CFT) were upgraded to accommodate finer granularity, and to facilitate improved downloads and diagnostics. The finer granularity improved fake track rejection, resulting in reduced Level 1 trigger rates. In addition, new electronics were installed to allow matching between signals from calorimeter trigger objects and charged tracks at Level 1. Technical commissioning of this Level 1 Calorimeter Track Match trigger was in progress at the end of FY 2006. The Level 2 trigger system was upgraded to increase available processing power. Finally, the online systems were upgraded to enhance performance and reliability, and the data acquisition system was upgraded to increase the rate of events that can be written to mass storage by more than a factor of two.

The DZero silicon microstrip tracker (SMT) provides the precision tracking and secondary vertex tagging capability essential to the DZero physics program. To take full advantage of the substantial integrated luminosities anticipated, an additional layer of silicon detectors was designed to fit between the beampipe and the silicon detectors that were installed prior to the start of Run II. This additional layer of silicon sensors, referred to as Layer 0 , provides additional hits on tracks, as well as improved impact parameter resolution. The design of this detector was based upon research and development performed for the larger, original Run IIb silicon detector upgrade. For example, the Layer 0 detector uses SVX4 readout chips 
originally designed and fabricated for the Run IIb silicon detector. The Layer 0 detector was prototyped, fabricated, and tested at the Fermilab Silicon Detector Facility. Since the radial clearance available for Layer 0 is less than $1 \mathrm{~mm}$, the detector installation had to be carefully planned and executed. Specialized tooling and procedures were developed and tested in various mock-ups. The Layer 0 detector installation, which required great care and attention to detail, was successfully performed during the 2006 shutdown. The duration of that shutdown was originally determined by the assessment of the time required for the Layer 0 installation. The Level 2 Silicon Track Trigger, which relies on inputs from the silicon microstrip tracker, was also upgraded to incorporate the information generated by the Layer 0 detector.

The CFT has performed well, and physics analyses are making good use of the enhanced tracking capabilities of the Run II tracking detectors. However, the performance of the Analog Front End boards (AFE) used to instrument the Visible Light Photon Counter readout of the CFT is expected to degrade as the instantaneous luminosity increases. Due to the high occupancies that will be encountered in the CFT channels, the readout electronics will saturate, resulting in reduced efficiency. In addition, the electronics exhibits undesirable channel-to-channel and crossing-to-crossing pedestal dependence, effectively forcing an unfortunate choice between reducing performance and increasing noise occupancy (and readout deadtime). To address these features, the AFEII readout board was developed. The AFEII is based upon a new readout chip that was designed specifically for this purpose. This readout chip also provides the capability to measure the time of arrival of the photons from the CFT. These timing measurements could be used to suppress accidental clusters during track reconstruction. Design, fabrication and testing of prototype and pre-production AFEII boards were completed during the fiscal year. Several of the pre-production AFEII boards were tested on the detector during the 2006 shutdown, and AFEII production was completed during September. A small number of the production AFEII boards were installed on the detector, and commissioning was in progress by the end of the fiscal year.

DZero had a substantial list of activities to complete during the 2006 shutdown (some of which are mentioned above), and those tasks were completed on time. The start-up of the accelerator complex and the DZero detector after this challenging shutdown went remarkably smoothly and efficiently. (Figure 3 illustrates the detector efficiency as a function of time.) DZero quickly re-established smooth operations, with almost all of the upgrades integrated into routine operations. Peak luminosities grew rapidly after the 2006 shutdown, and DZero has been refining a new trigger list (which implements the trigger upgrades) to effectively handle the increased instantaneous luminosity. 
B.9 Daily Data Taking Efficiency

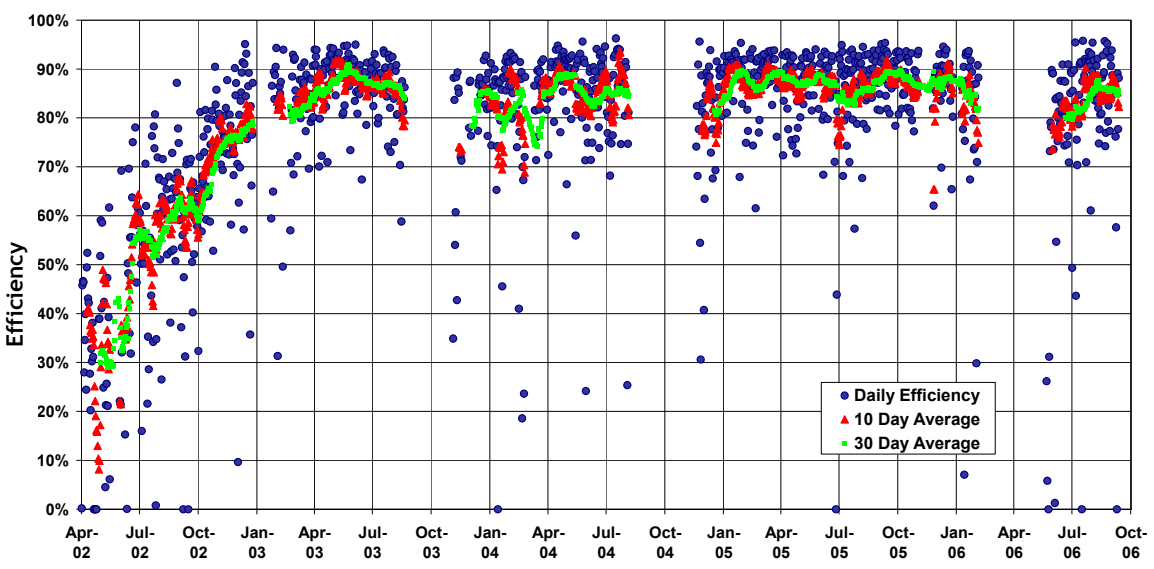

Figure 3. DZero data-taking efficiency as a function of time during Run II. The blue dots represent a daily average, the red triangles represent a 10-day average, and the green squares represent a 30 -day average.

As of October 20, 2005, DZero switched the default luminosity monitor readout from NIM based electronics, which utilized two discriminated signals produced from analog-summed signals from the 24 counters in each of the two detectors, to custom VME electronics with independent readout, discrimination, and monitoring of each of the 48 luminosity counter signals. This more detailed information allowed improved modeling and understanding of the detector acceptance, and coupled with the enhanced detector capabilities, facilitated a reassessment of the acceptance of the luminosity monitor and the associated luminosity constant, resulting in the approximately $12 \%$ upward revision (relative to the previously reported on-line numbers) of the luminosity values incorporated into this report.

During FY 2006, DZero recorded a total of $557 \mathrm{pb}^{-1}$ of the $655 \mathrm{pb}^{-1}$ of delivered integrated luminosity, which corresponds to an average efficiency of $85.0 \%$. Note that during FY 2006, the total recorded Run II data exposure increased by $50 \%$ to $1.66 \mathrm{fb}^{-1}$ (as illustrated in Figure 4).

\section{B.8 Run II Integrated Luminosity}

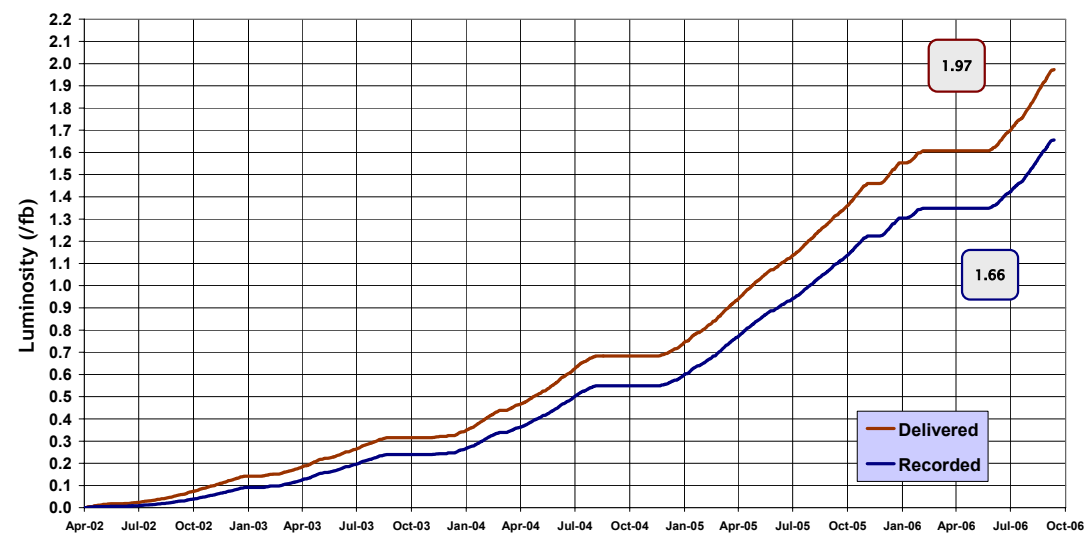

Figure 4. Delivered (red line) and recorded (blue line) luminosity at DZero as a function of time during Run II. 
NuMI / E-875 Main Injector Neutrino Oscillation Search (MINOS) (C. James, R. Plunkett)

\section{$\underline{N u M I}$}

The NuMI beam facility operated well during FY 2006, delivering its goal of $1 \times 10^{20}$ protons to the NuMI target between October 1, 2005 and September 30, 2006. Figure 5 shows the protons delivered per week during FY 2006, and Figure 6 shows the integrated protons during the same period. The down-times, and their cause, are noted in Figure 5, and explained further below.

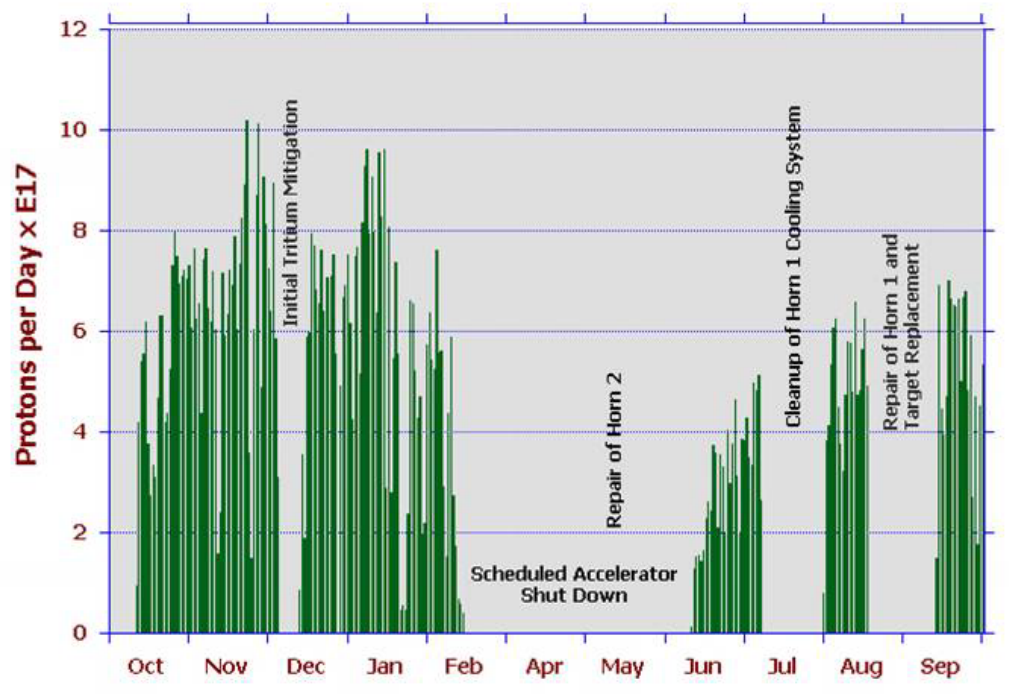

Figure 5. Protons per day on the NuMI target during FY 2006.

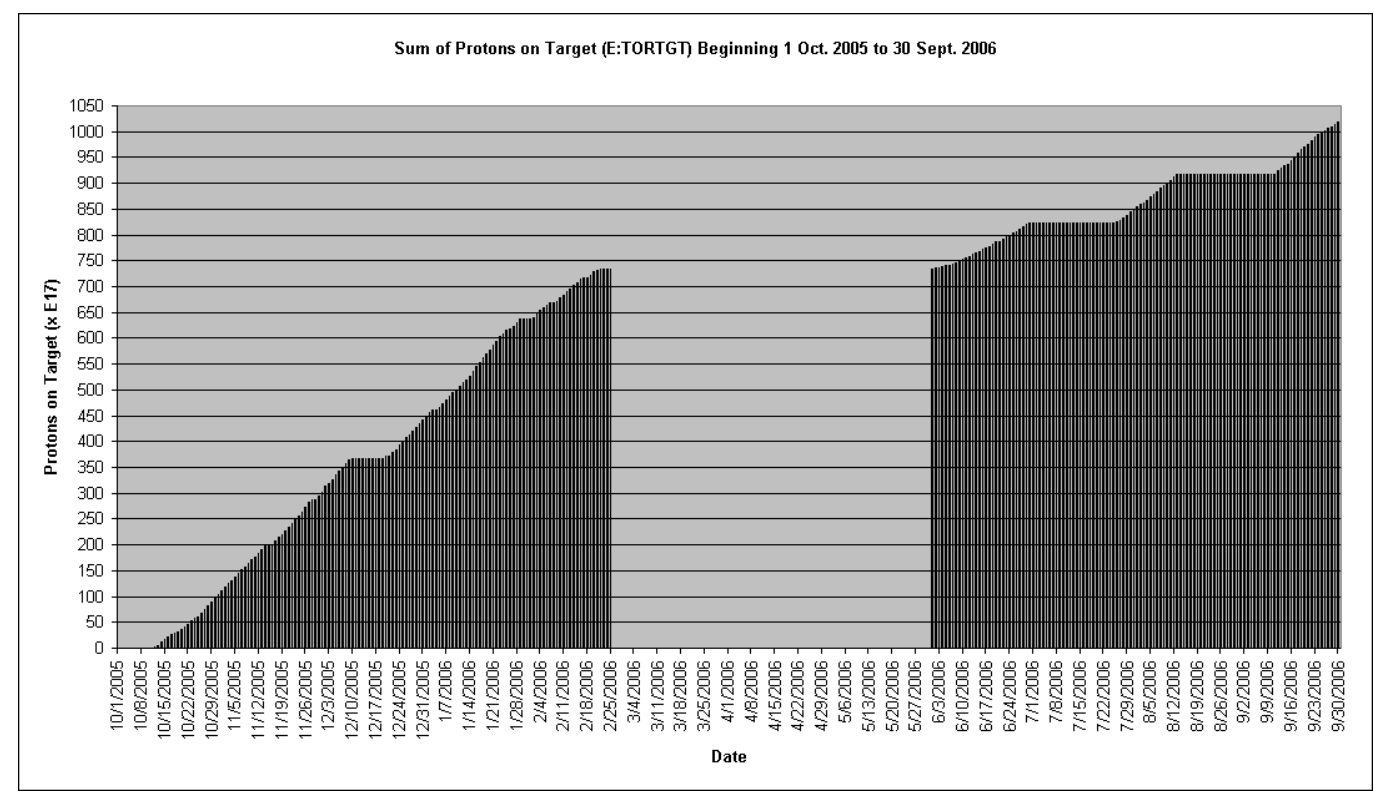

Figure 6. Integrated protons on the NuMI target for FY 2006. 
The two NuMI focusing horns are devices designed to withstand the mechanical shock from electrical pulsing and the stress on the materials due to beam exposure, but they do have a finite lifetime and their eventual failure and replacement is expected. By the end of September 2006 the horns had accumulated about 9.5 million pulses, which is $95 \%$ of the design goal, and $840 \mathrm{MW}$-hours of beam power.

The Target Hall and the Target Chase environment has proven to be hard on the equipment located there. A re-circulating air system is used to cool the Chase and the devices within it: the horn modules, the stripline which carries pulsed electrical current to the horns, the baffle, target and their carrier module, and the surrounding shielding (the horns themselves have a separate water-cooling system). The circulating cooling air picks up humidity from the underground environment, and exposure to beam ionizes the air. Subsequent chemistry produces ozone and nitric acid. The circulating air, passed through dehumidifiers, produces water with a $\mathrm{PH}$ of 2.6. This has caused varying levels of corrosion, depending on the material and its location.

Tritium is also produced in this environment. The Target Chase and the recirculating air system, while sealed, do have a small leakage rate, and a few percent of the circulating cooling air volume mixes into the air of the Target Hall. A variety of chemical pathways produce tritiated water, and some of this activated water ended up in the sump at the base of the MINOS shaft, where it was detected at higher than expected levels in December 2005. There has been much work done on sampling materials and modeling the various pathways which could lead to the amounts of tritium found in the various underground areas; and this has resulted in a better understanding of the process outlined above. Mitigation was installed, in the form of additional de-humidifers located in the Target Hall, which has removed a quantity of the activated water. Tritium levels found in the MINOS sump have since been reduced.

Corrosion effects led to the replacement of the target and target assembly in September 2006. While MINOS was finishing a special data period, where the target was moved to positions which produce higher neutrino beam energy spectra, it was found that the target would not return to the nominal low-neutrino beam energy location via remote control. One of the limit switches, used to prevent running the target into the horn, was also non-functional. A spare target and carrier assembly was ready, and the decision was made to install them.

Both horns required repairs during FY 2006, and both for the same reason, a leak in the horn cooling water supply lines. More specifically, a short portion of the piping, which is electrically-insulating ceramic, cracked and leaked. Replacing these ceramic parts would, in ordinary circumstances, be simple. However their location alongside the horn makes them and their surrounding environment highly activated. The repair process was identical for both horns. They were removed to the Work Cell located within the Target Hall, built for exactly this purpose, and, using tools and techniques developed using mock-ups, the ceramic parts were cut out and replaced. The repairs were well executed, and the absorbed dose to workers was well within Fermilab limits. Because of the level of activation, the repairs represent the limit of the work which can be performed. Any more serious damage would require the replacement of the entire horn. 
Horn 2 required this repair first, having started to leak in February 2006. The repair occurred during the scheduled accelerator shutdown period. Horn 1 started showing signs of the same leak in July. A preliminary investigation led to an accidental blockage of the Horn 1 water cooling system, which took three weeks in July to clear. In September the leak reached the point of requiring repair.

Construction of spares of both horns was in progress during the entire fiscal year and both spare horns are expected to be completed by early 2007. The group of people building the spares has been augmented by the addition of staff. Lessons learned from both the failures which occurred, and from observations of equipment during accesses, are being applied where possible to all the spare parts as they are being constructed. A notable example is a change to the type of ceramic insulator used in the horn water piping, and the manner in which the ceramics are installed in the piping.

Despite the difficulties in operating a high-intensity beamline, the NuMI facility has performed well. The target and horn system produced the desired neutrino spectrum for the MINOS experiment, and that neutrino spectrum was very stable over time, as shown in Figure 7. A review of the main items which caused problems during the fiscal year also reveals the items which worked well and according to design:

- The primary beam transport, the Autotune program, and the NuMI Beam Permit system functioned well, and as designed.

- The beam-to-horn pulse timing was within specifications on the very first extracted pulse of protons, and remained stable over the entire running period.

- At installation, Horn 1, Horn 2, and the Target were aligned by survey on the design beamline, and their locations confirmed by beam scans. The initial scans, and subsequent scans, utilized the horn "cross-hairs" and the beam monitors at the downstream end of the decay pipe, and all functioned well in this task.

- The horn motor drives functioned well, and as designed.

- The remote water connections for the horn and target cooling systems, and the remote strip-line connections, functioned well, and as designed.

- The shielding and remote handling systems for removing and replacing shielding functioned well, and as designed.

- The recirculating air system, once balanced, functioned well in its purpose as a cooling system. Measurements of the flow through each of the horn strip-line penetrations, around module walls, around the target, and through the shielding pile return path, are all as designed.

- The measured vibration of horns, hanging from their modules, was found to be small, as intended by the support design.

- Residual radiation rates in the Target Hall areas were approximately as predicted by modeling, which is important for planning repairs. 


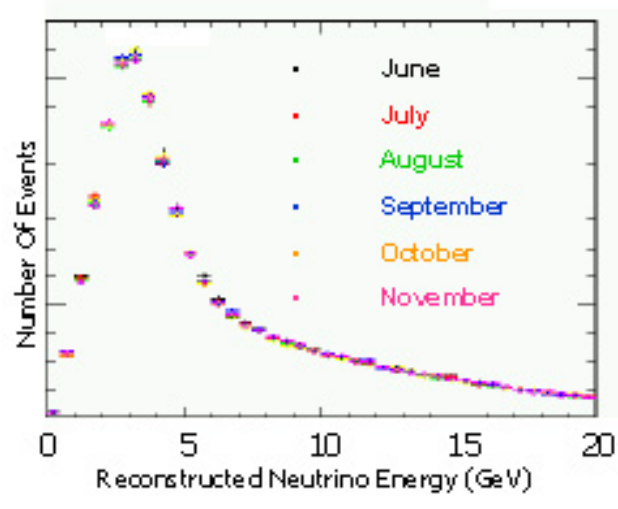

Neutrino Spectrum by Month

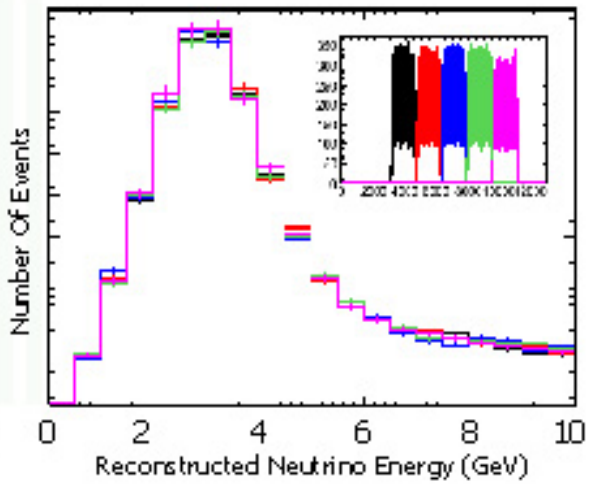

Neutrino Spectrum by Batdh

Figure 7. Stability of the produced neutrino energy spectrum of the NuMI beam.

$\underline{\text { MINOS }}$

The MINOS experiment operated very well during FY 2006. Both the Near and Far Detectors operated at high efficiency whenever beam was being delivered, always being active at least $95 \%$ of the time beam was delivered, and frequently above $98 \%$ of that time. The neutrino spectrum by month, shown in Figure 7, displays the stability of the NuMI beam. It also shows the stability over time of the Near Detector data recording and reconstruction, which was used to produce the plot. Both detectors have low rates of failure of electronic readout components. The supply of spares, and the repair of removed components for return to the spares pool, easily kept up with the replacement rate. No major problems emerged in the operation of either detector.

The data output from the detectors is processed on Fermilab computing farms. The processing keeps up with data production. The MINOS data, both raw and reconstructed, is stored in the Fermilab Computing Division's Enstore system, and the allocation is sufficient. Monte Carlo event generation is also performed on the Fermilab computing farms, and at a few MINOS collaborating institutions. The current amount of Monte Carlo data producing capacity is adequate.

Data collected between May 2005 and February 2006, representing $1.27 \times 10^{20}$ protons on target, was analyzed and the results of the neutrino oscillation measurement reported at Fermilab seminars and at various conferences between June 2006 and the present. This report will not describe the results further, except to include the contour plot of neutrino parameters showing the MINOS results compared to Super-K and K2K (Figure 8). 


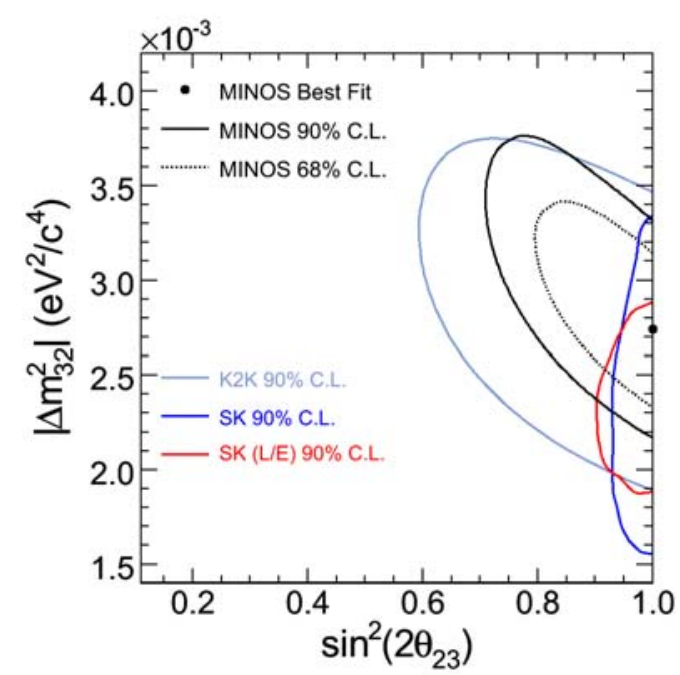

Figure 8. Contour plot of neutrino parameters showing MINOS results compared to Super-K and K2K.

$\underline{\text { E-898 / MiniBooNE (J. Conrad, W. Louis) }}$

The MiniBooNE E-898 experiment began taking data at Fermilab in late August, 2002. This section summarizes the MiniBooNE E-898 data collection and analysis progress during the 2006 fiscal year.

The Booster delivered about $7 \times 10^{20}$ protons to the MiniBooNE target during the four years of MiniBooNE E-898 operation, with about $0.5 \times 10^{20}$ protons in FY 2006 alone. The new focusing horn (replaced in 2004) has, so far, recorded over $100 \mathrm{M}$ pulses and has performed flawlessly.

Due to the short duty factor of the Booster beam, it is very easy to identify genuine neutrino events. Figure 9 shows the time distribution of events (in a 19 micro-second time interval around the Booster beam spill) that satisfy the criteria of more than 200 tank phototube hits and fewer than six veto phototube hits. As can be seen in the figure, most of the events are neutrino events produced during the 1.6 micro-second beam spill, and there are very few cosmicray-induced events outside the beam spill window. The ratio of neutrino events to cosmic-rayinduced events during the beam spill is about 5000 to 1 . Using the above criteria to identify neutrino events, MiniBooNE E-898 collected about $740 \mathrm{~K}$ neutrino events during the four years of data-taking, and approximately 60K neutrino events in FY 2006 alone. 


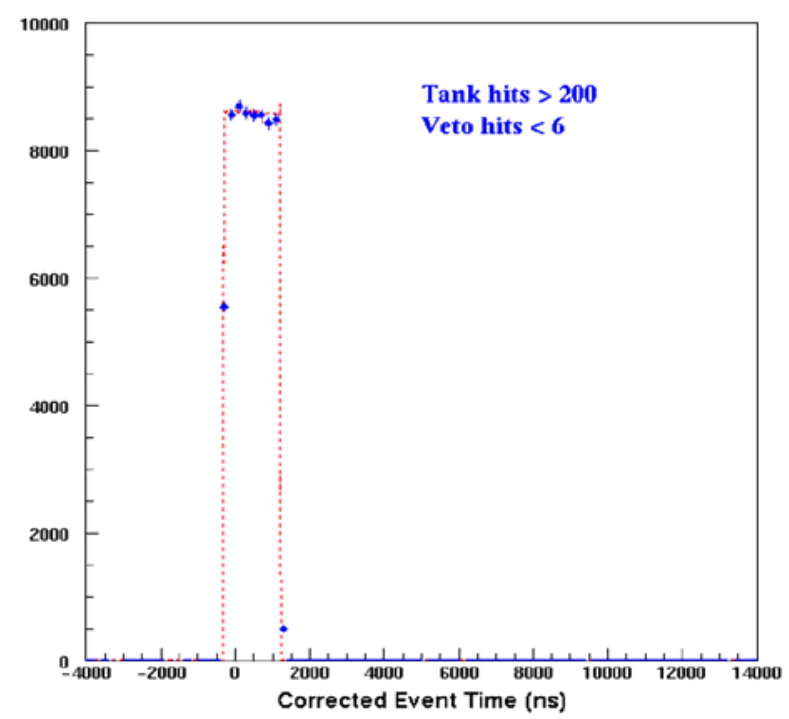

Figure 9. The time distribution of events (in a 19 micro-second time interval around the Booster beam spill) that satisfy the criteria of more than 200 tank phototube hits and fewer than 6 veto phototube hits.

The MiniBooNE detector and beamline also performed extremely well. In FY 2006, about $99 \%$ of the phototube channels worked well, and the data acquisition livetime averaged $\sim 99 \%$. Furthermore, the reconstructed time, position, energy, and angular resolutions are all in agreement with expectations; and the experiment is clearly observing charged-current quasielastic events, charged-current pion events, neutral-current pion events, and neutral-current elastic-scattering events. As an example, Figure 10 shows preliminary MiniBooNE chargedcurrent inclusive distributions for events containing a primary event and a secondary Michel electron. Good agreement is observed between the data (points with error bars) and Monte Carlo simulation (histogram) for the visible energy of the primary event, the number of tank hits of the primary event, the number of tank hits of the secondary event, and the radius of the primary event. Similarly, Figure 11 shows the particle identification parameter for charged-current inclusive events, where this parameter is the boosted-decision-tree output. 
cc data(black), dirtmc(red), 2 SE, thits $>200$, vhits $<6, R<500 \mathrm{~cm}$
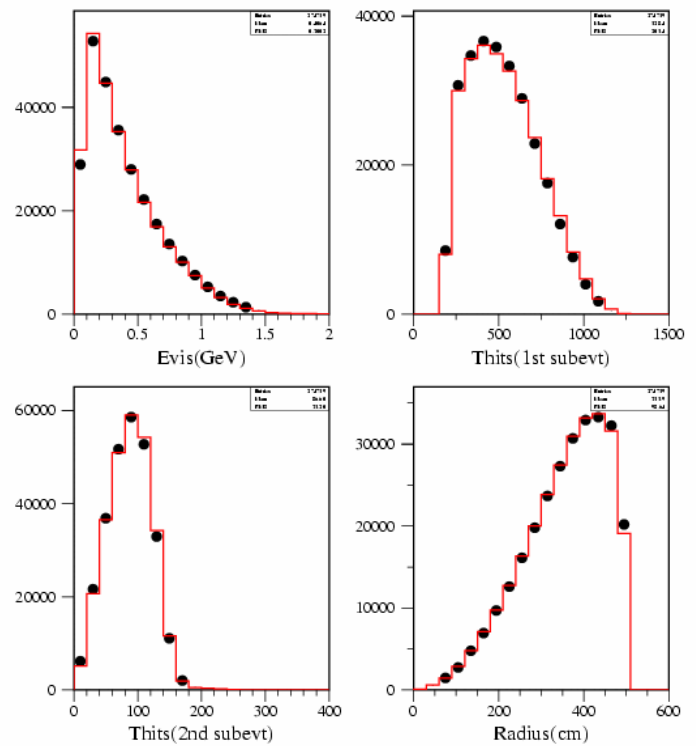

Figure 10. Preliminary MiniBooNE E-898 charged-current inclusive distributions for events containing a primary event and a secondary Michel electron.

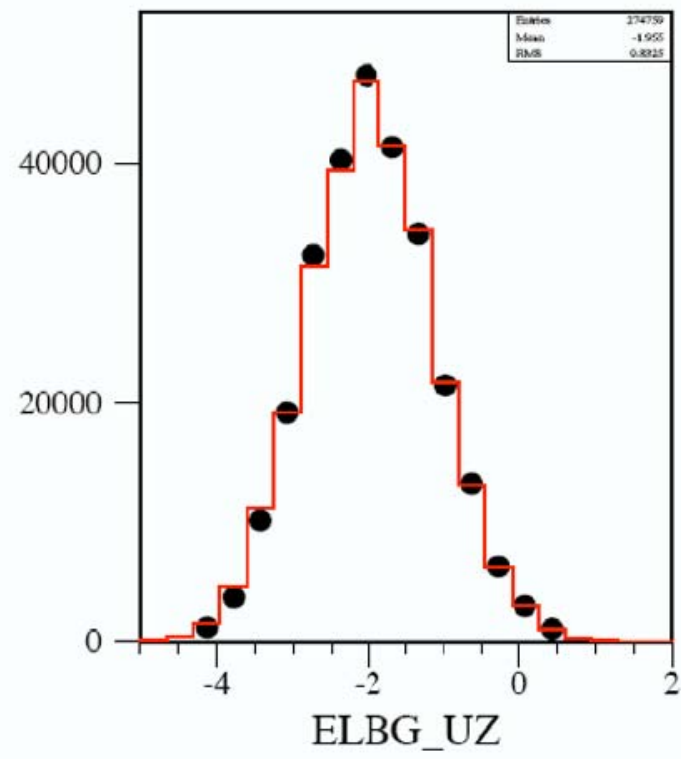

Figure 11. The particle identification parameter for charged-current inclusive events. 
Good agreement is again obtained between the data and Monte Carlo simulation. In addition, MiniBooNE is observing events produced by the NuMI beamline. Whereas the MiniBooNE neutrino event rate from the $8 \mathrm{GeV}$ beamline is about $1.1 \times 10^{-15}$ events per proton on target (POT), the event rate from the NuMI beamline is about $0.51 \times 10^{-15}$ events per POT.

Overall, 2006 was another good year of data-taking for the MiniBooNE E-898 experiment. The detector and beamline performed well, and the Booster delivered $0.5 \times 10^{20}$ POT to the experiment, despite the fact that data-taking stopped in January, 2006, when the focusing horn was switched to negative polarity for antineutrino running.

\section{$\underline{\text { E-944 / MiniBooNE (S. Brice, R. Van de Water) }}$}

Starting in early January 2006, a two-week shutdown of MiniBooNE operations occurred to swap out beam positive charging-supplies, and replace them with negative supplies. Work also was done on the power bus to make future change-outs easier and quicker, reducing a twoweek job to less than one week. The power supply change-out was successful, and on January 18, 2006 MiniBooNE E-944 began operations with a reversed horn current of -175kA, focusing negative charged particles, which preferentially produce antinuetrinos with a roughly $30 \%$ admixture of neutrinos.

Figure 12 shows the weekly performance since the start of MiniBooNE running in 2002. The antineutrino running is shown after the solid line; the large gap is the 2006 March shutdown which lasted three months. The performance of the Booster after the shutdown took some time to ramp up; but during August the Booster beam reached the "champagne goal" of $9 \times 10^{16}$ protons/hr for one hour, and the $1.08 \times 10^{19}$ protons delivered in that week broke the all-time record for weekly-integrated protons for neutrino production.

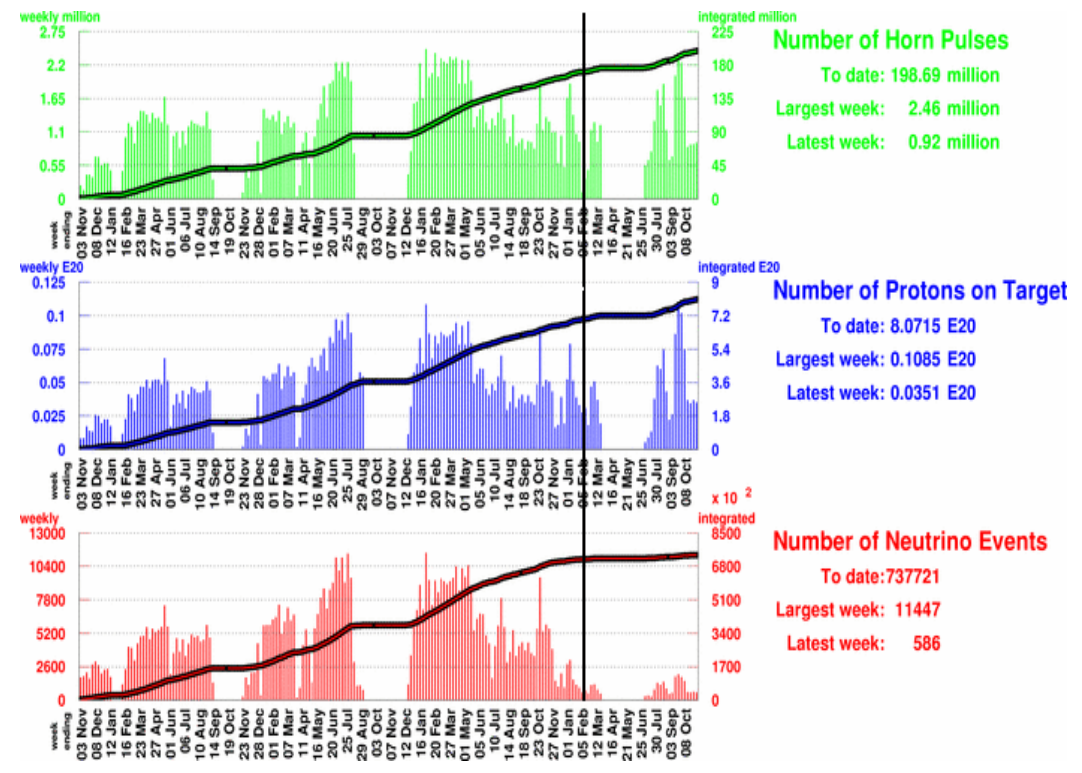

Figure 12. Weekly beam summary since the start of MiniBooNE operations in 2002. Antineutrino running is shown to the right of the solid vertical line. 
These milestones were due to the outstanding work of the Accelerator Division pushing the Booster to peak performance. This resulted in an impressive total of $1.1 \times 10^{20}$ protons on target (POT) delivered to MiniBooNE in the six months of beam since the start of antineutino running in January 2006. The overall performance is shown in Figure 13, which is a plot of the integrated protons-on-target delivered to the MiniBooNE beamline from January 18, 2006 to October 10, 2006.

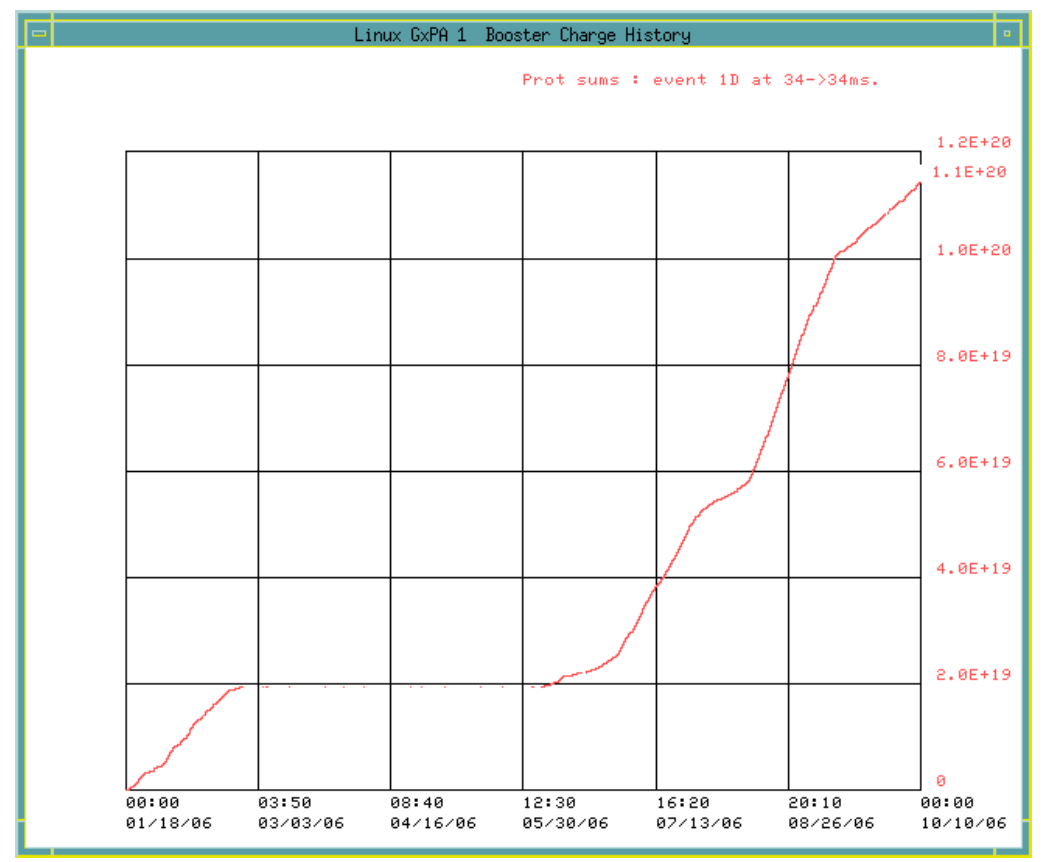

Figure 13. Integrated beam POT delivered to MiniBooNE from the start of antineutrino running Jan 18, 2006.

Over half the integrated data was collected while NuMI was down, which increases the instantaneous rates by about a factor of three. This can be seen in Figure 12 as two highintensity bumps in the POT plot. During normal running, i.e. NuMI on, a weekly average of about $0.3 \times 10^{19}$ POT is expected. This translates into $\sim 0.1 \times 10^{20}$ integrated POT per month, which implies ten months of running is needed to collect an extra $1 \times 10^{20}$ POT, taking E-944 to the planned start of the accelerator complex shutdown in June 2007. This would provide a total of $2 \times 10^{20}$ POT as desired for antineutrino cross section and disappearance oscillation measurements.

The bottom plot in Figure 12 demonstrates that the neutrinos collected per week are reduced by a factor of five relative to that of neutrino mode. This is expected from reductions in both flux and cross sections.

An important aspect to continued running of MiniBooNE is the reliability of the beamline, horn, and detector systems. During the antineutrino run period, the MiniBooNE uptime was about $98 \%$. This is for the combination of the beamline, horn, and detector. The most crucial element, the horn, has collected over 100 million pulses, surpassing the first horn, which failed two years ago with 94 million pulses (both of these horns have reached world records for most pulses). This second horn continues to run flawlessly with no signs of problems. 
In the unfortunate event of failure, there is a ready spare third horn and target. This would require three weeks to swap in, which would only be a small loss in total data.

To determine if the antineutrino event rates observed are in agreement with what is expected, a scale factor is computed that is the ratio of data rates to the current Monte Carlo simulation. The measured antineutrino scale factor is approximately unity for $0.7 \times 10^{20}$ POT. Figure 14 shows this via a plot of tank charge, which has a strong correspondence with energy, for both data and Monte Carlo, absolutely normalized. The good agreement demonstrates that the observed antineutrino inclusive rates are consistent with expectation, and that the data is of good quality.

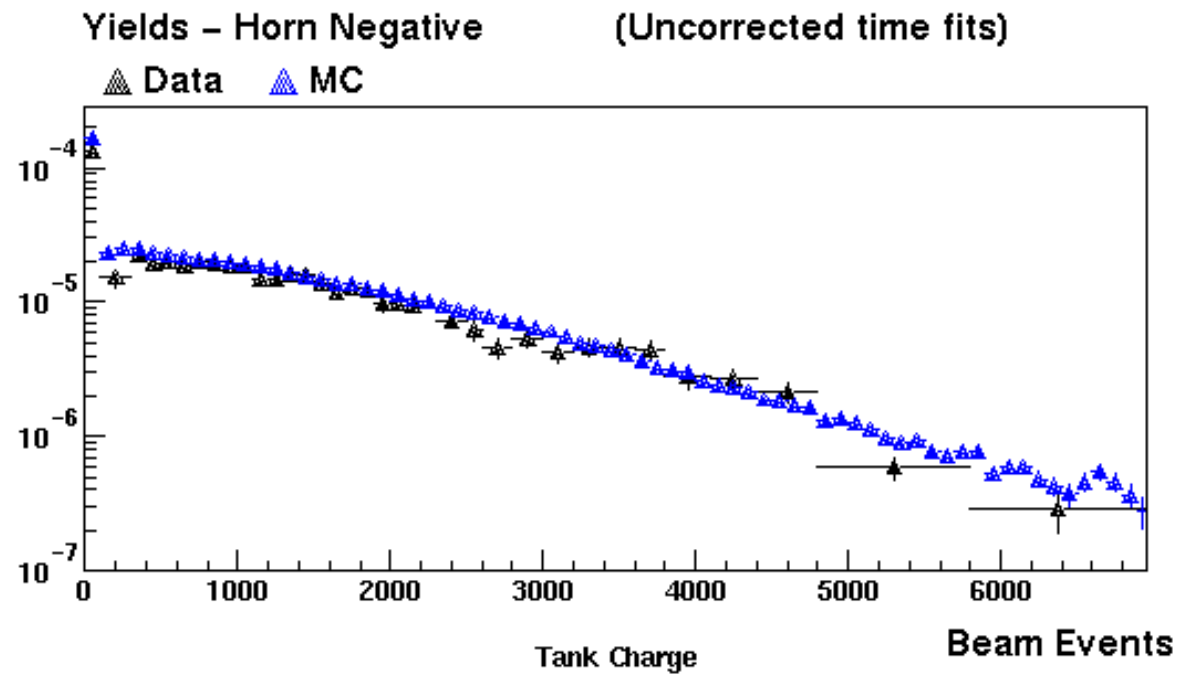

Figure 14. Data and MC detector tank charge (photoelectrons) absolutely normalized.

\section{Fixed-Target Switchyard 120 GeV (SY120) (C. Moore)}

SY120 had a productive year, with differing primary intensities and many different momentum tunes in the secondary beam portions of both beamlines: MTest and E-907 MIPP (Main Injector Particle Production). The primary intensity was changed by varying the number of turns and Booster bunches (from 2 turns, 12 bunches to 3 turns, 80 bunches). MTest ran with at least the following different momenta: $2 \mathrm{GeV} / \mathrm{c}, 3 \mathrm{GeV} / \mathrm{c}, 4 \mathrm{GeV} / \mathrm{c}, 8 \mathrm{GeV} / \mathrm{c}, 16 \mathrm{GeV} / \mathrm{c}$, $33 \mathrm{GeV} / \mathrm{c}, 66 \mathrm{GeV} / \mathrm{c}, 120 \mathrm{GeV} / \mathrm{c}$ and $-66 \mathrm{GeV} / \mathrm{c}$. MIPP ran with at least the following different momenta: -20 GeV/c, -30 GeV/c, -59 GeV/c, -85 GeV/c, $13.5 \mathrm{GeV} / \mathrm{c}, 15 \mathrm{GeV} / \mathrm{c}, 16.5 \mathrm{GeV} / \mathrm{c}, 17$ $\mathrm{GeV} / \mathrm{c}, 17.5 \mathrm{GeV} / \mathrm{c}, 18 \mathrm{GeV} / \mathrm{c}, 20 \mathrm{GeV} / \mathrm{c}, 30 \mathrm{GeV} / \mathrm{c}, 40 \mathrm{GeV} / \mathrm{c}, 50 \mathrm{GeV} / \mathrm{c}, 60 \mathrm{GeV} / \mathrm{c}, 75 \mathrm{GeV} / \mathrm{c}$, $85 \mathrm{GeV} / \mathrm{c}$, and $120 \mathrm{GeV} / \mathrm{c}$. In order to maximize the data-taking rate for MIPP, a nominal 4second spill was utilized. 
Number of Beam Pulses delivered to SY120 in FY06

The following number of pulses were tabulated when the critical device to the area was on and there was an appropriate event in the accelerator controls time line.

\begin{tabular}{|l|r|r|}
\hline & MIPP & MTest \\
\hline Total Fixed Target pulses & 143000 & 160000 \\
\hline Total nobeam pulses & 74000 & 113000 \\
\hline Total pulses with beam & 69000 & 47000 \\
\hline
\end{tabular}

\section{E-907 / MIPP - Main Injector Particle Production (R. Raja)}

The MIPP experiment (E-907) was designed to measure particle production using Main Injector primary and secondary beams $\left(\pi^{ \pm}, \mathrm{K}^{ \pm}, \mathrm{p}^{ \pm}\right.$with beam momenta ranging from $5 \mathrm{GeV} / \mathrm{c}$ to $90 \mathrm{GeV} / \mathrm{c}$ ) over a variety of targets ranging from hydrogen to beryllium, carbon and heavy nuclei. The MIPP apparatus is shown in Figure 15. The centerpiece of the experiment was a time projection chamber (TPC), capable of detecting particles over nearly $4 \pi$ acceptance. Using a combination of $\mathrm{dE} / \mathrm{dx}$, time of flight, Cerenkov, and $\mathrm{RICH}$ detectors, MIPP identified the charged particles in the final state. With the data taken, MIPP hopes to restart the study of nonperturbative QCD interactions as well as to measure cross sections in nuclei for the purposes of proton radiography, high-energy physics, and nuclear physics. A critical measurement MIPP will make is the measurement of particle production from the NuMI target, which will be beneficial to all neutrino experiments using the NuMI beam (MINOS, MINERvA, NOvA).

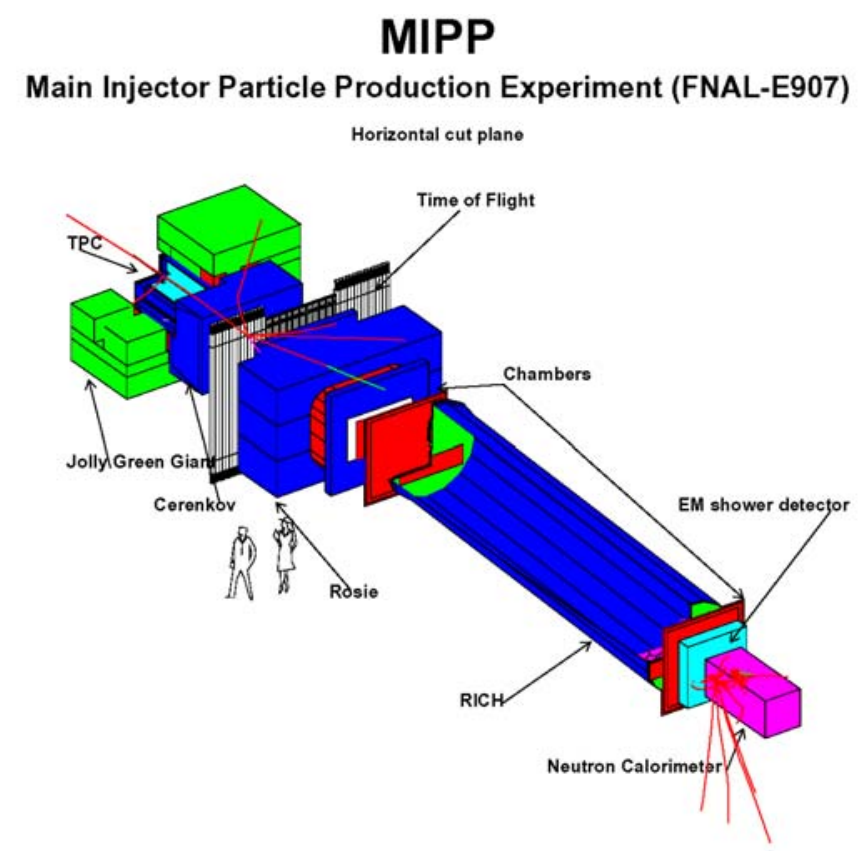

Figure 15. The MIPP apparatus. 
MIPP finished its data-taking run in February 2006, having run a year with the TPC data acquisition speed at an average of $\sim 25 \mathrm{~Hz}$. MIPP recorded $\sim 18$ million events in this mode. During the last month of the run, the Jolly Green Giant magnet coils having shorted out, MIPP switched off the TPC and took data at $\sim 300 \mathrm{~Hz}$ as a test of the MIPP ability to measure the charged kaon mass using the ring radii in the RICH. Table 1 gives the summary of the physics data taken by MIPP (FY 2005 and 2006) as a function of target and beam momentum.

Table 1. Summary of data taken during MIPP physics run.

\begin{tabular}{|c|c|c|c|c|c|c|c|c|c|c|c|c|}
\hline \multirow{2}{*}{\multicolumn{3}{|c|}{$\begin{array}{c}\text { Data Summary } \\
27 \text { February } 2006 \\
\text { Target }\end{array}$}} & \multicolumn{10}{|c|}{$\begin{array}{l}\text { Acquired Data by Target and Beam Energy } \\
\text { Number of events, } \times 10^{6}\end{array}$} \\
\hline & & & \multicolumn{9}{|c|}{$\mathbf{E}$} & \multirow[b]{2}{*}{ Total } \\
\hline $\mathbf{Z}$ & Element & $\begin{array}{l}\text { Trigger } \\
\text { Mix }\end{array}$ & 5 & 20 & 35 & 40 & 55 & 60 & 65 & 85 & 120 & \\
\hline \multirow{3}{*}{$\mathbf{0}$} & Empty & Normal & & 0.10 & 0.14 & & & 0.52 & & & 0.25 & 1.01 \\
\hline & $K$ Mass & No Int. & & & & 5.48 & 0.50 & 7.39 & 0.96 & & & 14.33 \\
\hline & Empty LH & Normal & & 0.30 & & & & 0.61 & & 0.31 & & \multirow{2}{*}{7.08} \\
\hline $\mathbf{1}$ & LH & Normal & 0.21 & 1.94 & & & & 1.98 & & 1.73 & & \\
\hline \multirow{2}{*}{4} & \multirow{2}{*}{ Be } & $p$ only & & & & & & & & & 1.08 & \multirow{2}{*}{1.75} \\
\hline & & Normal & & & 0.10 & & & 0.56 & & & & \\
\hline \multirow{3}{*}{6} & $\mathrm{C}$ & Mixed & & & & & & 0.21 & & & & \multirow{2}{*}{1.33} \\
\hline & C $2 \%$ & Mixed & & 0.39 & & & & 0.26 & & & 0.47 & \\
\hline & NuMI & $p$ only & & & & & & & & & 1.78 & 1.78 \\
\hline 13 & Al & Normal & & & 0.10 & & & & & & & 0.10 \\
\hline \multirow{2}{*}{83} & \multirow{2}{*}{$\mathbf{B i}$} & $p$ only & & & & & & & & & 1.05 & \multirow{2}{*}{2.83} \\
\hline & & Normal & & & 0.52 & & & 1.26 & & & & \\
\hline 92 & $\mathbf{U}$ & Normal & & & & & & 1.18 & & & & 1.18 \\
\hline \multicolumn{3}{|c|}{ Total } & 0.21 & 2.73 & 0.86 & 5.48 & 0.50 & 13.97 & 0.96 & 2.04 & 4.63 & 31.38 \\
\hline
\end{tabular}

\section{Meson Test Beam Facility (E. Ramberg)}

The Meson Test Beam Facility (MTBF) continued regular operations during FY 2006. This facility gives users from around the world an opportunity to test the performance of their particle detectors in a high energy beam. A plan view of the facility is shown in Figure 16. The web site for the facility can be found at http://www-ppd.fnal.gov/MTBF-w/ 


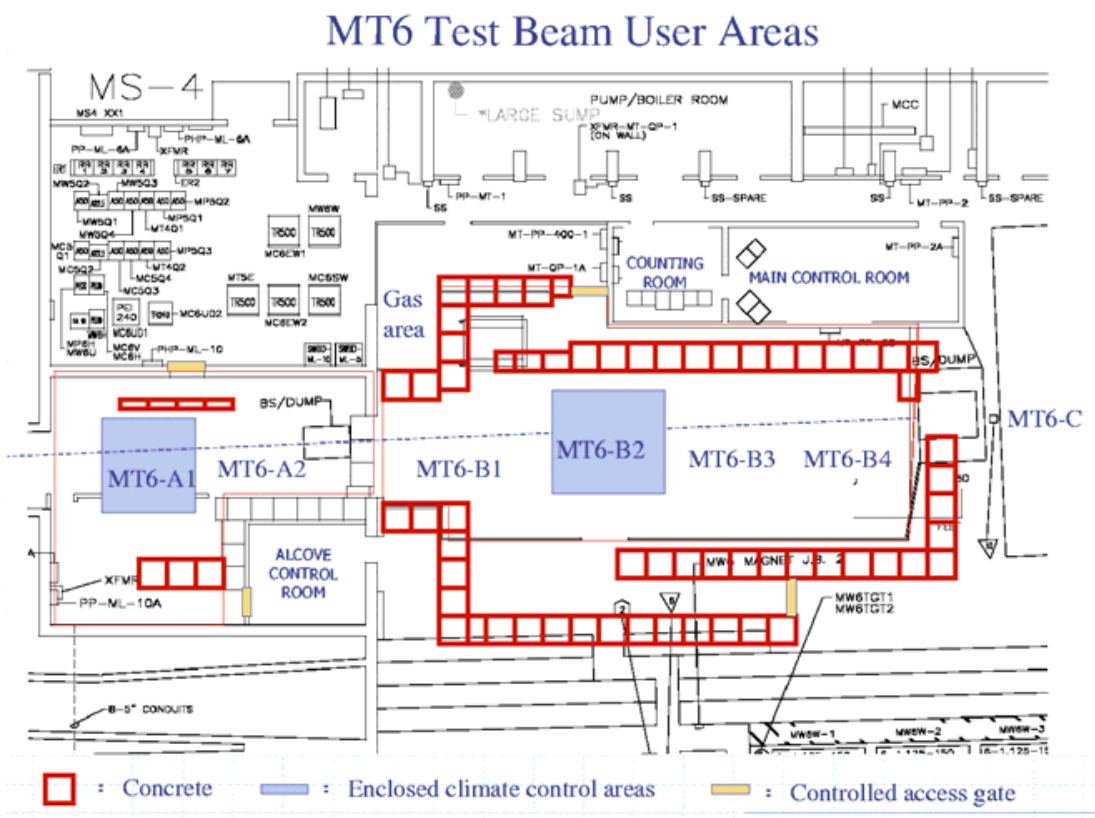

Figure 16. A plan view of the Meson Test Beam Facility.

The test beam is part of the Switchyard 120 (SY120) program, and uses resonant extraction of at least one Booster batch inside the Main Injector (MI). This batch usually consists of 60-80 RF 'buckets', with bucket separation of 19 nsec. Thus the batch is about 1.5 microseconds long. The full circumference of the MI is about 10 microseconds. It is possible to run with up to five Booster batches in the MI. The batch or batches are accelerated to $120 \mathrm{GeV}$, circulate around the MI, and are slowly extracted over a macroscopic slow spill. For most usage of the test beam, there was less than $100 \mathrm{kHz}$ of beam. If beam were smoothly extracted, this would imply that at most one particle per rotation of the MI batch would be extracted. The beam is not that smooth, and there is up to $35 \%$ double occupancy per MI rotation.

The length and duty cycle of the spill is determined by the Accelerator Division, with guidance from Program Planning. For most of FY 2006, the beam spill was 3.6 seconds long, one spill per minute for 12 hours per day. In summer 2006 the Accelerator Division established a 0.6 second spill for about a week before returning to the 3.6 second spill. Currently it is difficult to switch from one spill length to the other, but it is expected that this capability will become a more routine operation.

The maximum beam intensity in the Main Injector that was extracted to the test beam target was approximately $2.4 \times 10^{12}$ per spill. When sending primary protons to users $(120 \mathrm{GeV})$, a pinhole collimator is used to reduce rates for safety. This translates into 800,000 protons per spill at $120 \mathrm{GeV}$. When tuned to the lowest secondary-beam momentum achieved so far (4 $\mathrm{GeV}$ ), the maximum particle rate is 500 particles per spill with no collimation. About half of these particles are electrons. Table 2 gives a summary of the rates and electron content of various test beam tunes. 
Table 2. Rate of beam in the MTBF user area (MT6) and fractional flux of electrons. All rates are normalized to $2.4 \times 10^{12}$ protons in the Main Injector.

\begin{tabular}{|c|c|c|}
\hline Tune $(\mathrm{GeV})$ & Total rate/spill & e-fraction \\
\hline & & \\
\hline 120 & 800,000 & 0 \\
\hline 66 & 90,000 & 0 \\
\hline 33 & 40,000 & $0.7 \%$ \\
\hline 16 & 15,000 & $10 \%$ \\
\hline 8 & 5,000 & $30 \%$ \\
\hline 4 & 500 & $60 \%$ \\
\hline
\end{tabular}

The beam has an approximately $1 \%$ momentum spread, and can be focused to a $7 \mathrm{~mm}$ rms spot size for $120 \mathrm{GeV}$ protons and approximately $2-5 \mathrm{~cm}$ rms spot for the lower momenta.

Each test beam experiment is required to develop a Memorandum of Understanding (MOU) with the Laboratory. In the MOU, the beam and infrastructure requirements are spelled out in detail. Five new tests were approved and took data during FY 2006, in addition to a test that took data starting in FY 2005. These tests are shown in Table 3.

Table 3. MTBF tests performed in FY 2006.

\begin{tabular}{|l|l|}
\hline Test & Description \\
\hline & \\
\hline T-936 & US/CMS Forward Pixel \\
\hline T-953 & U. Iowa Cerenkov Light Tests \\
\hline T-955 & RPC Detector Tests \\
\hline T-956 & ILC Muon Detector Tests \\
\hline T-957 & NIU Tail Catcher/Muon Test \\
\hline T-958 & FP420 Fast Timing Test \\
\hline
\end{tabular}

In September 2006, the MTBF shut down for significant upgrades to both the beamline and the infrastructure of the user areas. The beamline improvements include:

- Installation of a new target, closer to the user areas;

- Minimization of scattering material;

- Addition of low-current power supplies and Hall probes for the bending magnets; and

- Addition of several new quadrupole doublets for better focusing.

User infrastructure improvements include:

- New differential Cerenkov counter;

- Time-of-flight detector system;

- $\quad 4^{\text {th }}$ MWPC tracking station;

- Addition of new space in the control room;

- Epoxy painting of the MTBF floor; and

- Complete reinstallation of cabling between beamline and electronics room.

These improvements will be complete in December 2006. 